Louisiana State University

LSU Digital Commons

Faculty Publications

Department of Physics \& Astronomy

7-27-2007

\title{
Recoil velocities from equal-mass binary-black-hole mergers
}

\author{
Michael Koppitz \\ Max Planck Institute for Gravitational Physics (Albert Einstein Institute) \\ Denis Pollney \\ Max Planck Institute for Gravitational Physics (Albert Einstein Institute) \\ Christian Reisswig \\ Max Planck Institute for Gravitational Physics (Albert Einstein Institute) \\ Luciano Rezzolla \\ Max Planck Institute for Gravitational Physics (Albert Einstein Institute) \\ Jonathan Thornburg \\ Max Planck Institute for Gravitational Physics (Albert Einstein Institute)
}

See next page for additional authors

Follow this and additional works at: https://digitalcommons.Isu.edu/physics_astronomy_pubs

\section{Recommended Citation}

Koppitz, M., Pollney, D., Reisswig, C., Rezzolla, L., Thornburg, J., Diener, P., \& Schnetter, E. (2007). Recoil velocities from equal-mass binary-black-hole mergers. Physical Review Letters, 99 (4) https://doi.org/ 10.1103/PhysRevLett.99.041102

This Article is brought to you for free and open access by the Department of Physics \& Astronomy at LSU Digital Commons. It has been accepted for inclusion in Faculty Publications by an authorized administrator of LSU Digital Commons. For more information, please contact ir@lsu.edu. 


\section{Authors}

Michael Koppitz, Denis Pollney, Christian Reisswig, Luciano Rezzolla, Jonathan Thornburg, Peter Diener, and Erik Schnetter 


\title{
Recoil Velocities from Equal-Mass Binary-Black-Hole Mergers
}

\author{
Michael Koppitz, ${ }^{1}$ Denis Pollney, ${ }^{1}$ Christian Reisswig, ${ }^{1}$ Luciano Rezzolla, ${ }^{1,2}$ Jonathan Thornburg, ${ }^{1}$ \\ Peter Diener, ${ }^{3,2}$ and Erik Schnetter ${ }^{3}$ \\ ${ }^{1}$ Max-Planck-Institut für Gravitationsphysik, Albert-Einstein-Institut, Potsdam-Golm, Germany \\ ${ }^{2}$ Department of Physics and Astronomy, Louisiana State University, Baton Rouge, Louisiana 70803, USA \\ ${ }^{3}$ Center for Computation and Technology, Louisiana State University, Baton Rouge, Louisiana 70803, USA
}

(Received 29 January 2007; revised manuscript received 5 April 2007; published 27 July 2007)

The final evolution of a binary-black-hole system gives rise to a recoil velocity if an asymmetry is present in the emitted gravitational radiation. Measurements of this effect for nonspinning binaries with unequal masses have pointed out that kick velocities $\sim 175 \mathrm{~km} / \mathrm{s}$ can be reached for a mass ratio $\simeq 0.36$. However, a larger recoil can be obtained for equal-mass binaries if the asymmetry is provided by the spins. Using two independent methods we show that the merger of such binaries yields velocities as large as $\sim 440 \mathrm{~km} / \mathrm{s}$ for black holes having unequal spins that are antialigned and parallel to the orbital angular momentum.

DOI: 10.1103/PhysRevLett.99.041102

Introduction. -Binary-black-hole systems are expected to be one of the strongest sources of gravitational waves and are therefore the subject of intense and careful investigations. With Earth-based gravitational-wave detectors now working at design sensitivity and a space-borne detector in its formulation phase, the need for reliable templates to be used in matched filtering techniques has inspired a renewed enthusiasm in numerical analysis. Using numerical methods developed recently [1-3] there has been an explosion of results (see, e.g., Refs. [4-16]).

These developments are important for at least three different reasons. First, they allow for improved templates to be used in the analysis of the data coming from the detectors. Second, they allow probes of general relativity in regimes that have previously been inaccessible. Last but not least, they can provide, even through the solution of the Einstein equations in vacuum, important astrophysical information.

Together with energy and angular momentum, gravitational radiation also carries away linear momentum. In the case of a binary system of nonspinning black holes, a physical intuition of this loss of linear momentum can be built rather easily. As the two bodies orbit around the common center of mass, each will emit radiation which is forward beamed. Unless the two black holes have exactly the same mass, their motion will be different, with the smaller black hole moving more rapidly and, hence, being more efficient in beaming its emission. The net momentum gained over an orbit is negligible if the orbit is almost circular (the momentum loss in any direction is essentially balanced by an equal loss in the diametrically opposite direction), but it can become large when integrated over many orbits, leading to a recoil that is a fraction $\left(\$ 10^{-2}\right)$ of the speed of light during the last portion of the orbit prior to the merger.

A number of post-Newtonian (PN) or perturbative analyses (see, e.g., [17,18]) have provided estimates of this recoil velocity, while numerical-relativity simulations
PACS numbers: 04.25.Dm, 04.30.Db

$[8,11]$ have recently measured it to rather high precision, predicting a maximal kick of $175 \mathrm{~km} / \mathrm{s}$ for a binary system of nonspinning black holes with a mass ratio $q \equiv$ $M_{1} / M_{2} \simeq 0.36$, where $M_{1}$ and $M_{2}$ are the masses of the two black holes. Such a recoil has indeed quite important astrophysical consequences, since it could, provided it is large enough, kick the binary out of its host environment. Clearly, a replaced or an even missing central black hole would have dramatic consequences for the further development of the host. Determining accurately the expected escape velocities for the most typical environments hosting a binary-black-hole system is rather difficult, but the estimates made in Ref. [19], for instance, predict that the escape velocities for dwarf galaxies and globular clusters are $\$ 100 \mathrm{~km} / \mathrm{s}$, but for giant galaxies these can be $\sim 1000 \mathrm{~km} / \mathrm{s}$.

When adopting a purely geometrical viewpoint, it is obvious that a kick velocity should be expected in any binary system which is not perfectly symmetric. A difference in the masses is a simple way of producing such an asymmetry but surely not the only one. Indeed, even an equal-mass system can be made asymmetric if the two black holes have unequal spins. Also in this case, a simple physical intuition can be constructed. Consider, for simplicity an equal-mass binary in which only one member is spinning parallel to the orbital angular momentum. As a result of the spin-induced frame dragging, the speed of the nonspinning body will be increased and its radiation further beamed. Using PN theory at the 2.5 order, Kidder [20] has treated this spin-orbit interaction concluding that in the case of a circular, nonprecessing orbit, the total kick for a binary system of arbitrary mass and spin ratio can be expressed as [17]

$$
|v|_{\mathrm{kick}}=c_{1} \frac{q^{2}(1-q)}{(1+q)^{5}}+c_{2} \frac{a_{2} q^{2}\left(1-q a_{1} / a_{2}\right)}{(1+q)^{5}},
$$

where $a_{1,2} \equiv S_{1,2} / M_{1,2}^{2}$ are the dimensionless spins of the 
two black holes and these are aligned with the total orbital angular momentum, i.e., $\boldsymbol{S}_{1,2}=a_{1,2} M_{1,2}^{2} \boldsymbol{e}_{z}$ for an orbital motion in the $(x, y)$ plane. Here, $c_{1}$ and $c_{2}$ are factors depending on the total mass of the system and on the orbital separation at which the system stops radiating. This radius is difficult to determine precisely as it lies in a region where the $\mathrm{PN}$ approximation is not very accurate and is, in practice, not even a constant but, rather, depends on both the mass and the spin ratio. Assuming for simplicity $c_{1} \simeq c_{2}$, expression (1) reveals that a substantial contribution to the recoil velocity comes from the spins alone. In addition, for any given $q$, it predicts a linear growth of the recoil velocity with increasing difference in spins, yielding a kick which is comparable with the one coming from the asymmetry in the mass. Stated differently, when it comes to recoil velocities, the spin contributions may be the dominant ones.

Techniques. - The numerical evolutions have been carried out using a conformal-traceless formulation of the Einstein equations as described in [21], with " $1+\log$ " slicing and $\Gamma$-driver shift, and advection terms applied to the gauge conditions as suggested in [3]. Spatial differentiation is performed via straightforward finite differencing using fourth-order stencils. Individual apparent horizons are located every few time steps during the evolution [22]. Vertex-centered adaptive mesh-refinement is employed using nested grids [23] with the highest resolution concentrated in the neighborhood of the individual horizons. For each of the models studied, we have carried out simulations with fine-grid resolutions of $h=0.030 M$ and $h=$ $0.024 M$, where $M \equiv M_{1}+M_{2}$ is the total mass of the system. For a subset we have carried out a further evolution at $h=0.018 M$ to assess the convergence and estimate the error for the $h=0.024 M$ results.

The initial data are constructed using the "puncture" method [24], which uses Bowen-York extrinsic curvature and solves the Hamiltonian constraint equation numerically as in [25]. We have considered a sequence of binaries for which the initial spin of one of the black holes is fixed at $S_{2} / M^{2}=0.146 e_{z}$, and, to maximize the recoil [cf. Eq. (1)], the spin of the second black hole is in the opposite direction and has a modulus which is varied in steps of $1 / 4$. As a result, we obtain 5 sets of binary black holes having spin ratios $-1,-3 / 4, \ldots, 0$. The orbital parameters $\left(m_{i}, x_{i}\right.$, and $p_{i}$ ) for spinning binaries in quasicircular orbit are determined using an effective potential method [26] (a minimum is found in the binding energy of the system) in such a way that the black-hole masses $\left(M_{i}=\right.$ $\left.\sqrt{M_{\mathrm{ir}}^{2}+S_{i}^{2} /\left(4 M_{\mathrm{ir}}^{2}\right)}\right)$ are equal and $M=1$. The data for the initial separation and momenta are summarized in Table I and are chosen so that all of the binaries have the same orbital angular momentum.

To avoid possible systematic errors and improve the accuracy of the measurements, the kick velocity has been computed using two different and independent methods. The first and more traditional one makes use of the
TABLE I. The puncture initial data parameters defining the binaries: location $( \pm x / M)$, linear momenta $( \pm p / M)$, masses $\left(m_{i} / M\right)$, spins $\left(S_{i} / M\right)$, and Arnowitt-Deser-Misner (ADM) mass measured at infinity $\left(M_{\mathrm{ADM}}\right)$.

\begin{tabular}{cccccccc}
\hline \hline & $\pm x / M$ & $\pm p / M$ & $m_{1} / M$ & $m_{2} / M$ & $S_{1} / M^{2}$ & $S_{2} / M^{2}$ & $M_{\mathrm{ADM}} / M$ \\
\hline$r 0$ & 3.0205 & 0.1366 & 0.4011 & 0.4009 & -0.1460 & 0.1460 & 0.9856 \\
$r 1$ & 3.1264 & 0.1319 & 0.4380 & 0.4016 & -0.1095 & 0.1460 & 0.9855 \\
$r 2$ & 3.2198 & 0.1281 & 0.4615 & 0.4022 & -0.0730 & 0.1460 & 0.9856 \\
$r 3$ & 3.3190 & 0.1243 & 0.4749 & 0.4028 & -0.0365 & 0.1460 & 0.9857 \\
$r 4$ & 3.4100 & 0.1210 & 0.4796 & 0.4034 & -0.0000 & 0.1460 & 0.9859 \\
\hline \hline
\end{tabular}

Newman-Penrose quantity $\psi_{4}$ to provide the rate of change of the linear momentum in the $i$ direction as $[27,28]$

$$
\frac{d P_{i}}{d t}=\lim _{r \rightarrow \infty}\left\{\frac{r^{2}}{16 \pi} \int d \Omega \frac{x_{i}}{r}\left|\int_{-\infty}^{t} d t \Psi_{4}\right|^{2}\right\} .
$$

The second and novel method, instead, uses a perturbative wave-extraction procedure [29] that calculates gravitational waves in terms of gauge invariant, even- $Q_{\ell m}^{(e)}$ and odd-parity $Q_{\ell m}^{(o)}$ metric perturbations of the Schwarzschild spacetime after they have been decomposed into spherical harmonics of spin weight -2 [30]. Using these quantities it is possible to reconstruct the momentum fluxes in the $x$ and $y$ directions as

$$
\dot{P}_{x}+i \dot{P}_{y}=\sum_{\ell, m}^{\infty} F\left(Q_{\ell m}^{(e, o)}, Q_{\ell m}^{(e, o) \star}, \dot{Q}_{\ell m}^{(e, o)}, \dot{Q}_{\ell m}^{(e, o) \star}\right),
$$

where the overdot indicates a (coordinate) time derivative and the $\star$ indicates complex conjugation [the complete expression for the formula (3) will be presented in a longer paper [31]].

We have validated both methods by measuring the recoil velocity for a binary system of nonspinning black holes having a mass ratio of $2 / 3$ at an initial separation of $4.1 M$. The results of this calibration extracted at $r=50 \mathrm{M}$ are shown in Fig. 1, which reports the evolution of the kick velocity using $\Psi_{4}$ (solid lines), and the gauge-invariant quantities when the summation in (3) is truncated to the first 6 multipoles (dashed lines), which we have found to be sufficient to reach convergence. Indicated with symbols are the estimates and relative error bars obtained by [8] (circle) and by [11] (star).

We note that because the binary system starts evolving at a finite separation, it will have already gained a net linear momentum which can influence the value of the final kick. Computing this initial linear momentum amounts to selecting a proper constant in the integration of (2) or (3). Fortunately, this is rather straightforward to do and amounts to determining the direction in 3-space in which the center of mass of the system is moving initially. In practice, we plot the evolution of the $x$ and $y$ components of the kick velocity (the $z$ component is zero because of symmetry) and calculate the vector to the center of the spiral generated as the evolution proceeds. This vector is 


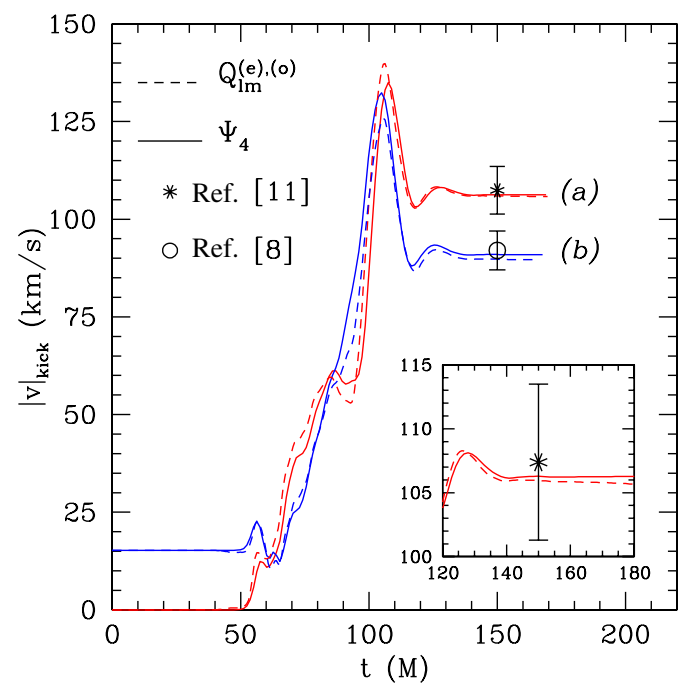

FIG. 1 (color online). Recoil velocity as function of time for a binary system of nonspinning black holes with a mass ratio of $2 / 3$ at an initial separation $4.1 \mathrm{M}$. The set of curves (a) and (b) differ in the choice of the integration constant, while the solid and dashed lines show the two independent computations of the momentum flux [Eqs. (2) and (3)].

then composed with the final one, yielding the final kick; note that being a vector this integration constant is not simply an additive constant for the kick velocity $|v|_{\text {kick }}$. In Fig. 1, we have plotted the effect of including this constant, comparing the case where it is set to zero [set of curves (a)], with a value set by extrapolating the recoil backwards to compensate for the small but nonzero initial linear momentum [set of curves (b)]. In the first case we find agreement with [11], while in the second case the good agreement is with [8].

A validation of this procedure is also rather straightforward: only an accurate estimate of the initial momentum yields a monotonic evolution of the kick velocity (or, in the case of very close binaries, reduces the oscillations considerably); any different choice would yield the oscillations seen in curves (a) (cf. Fig. 1 of Ref. [11] or Fig. 3 of Ref. [11]). Clearly, selecting the correct integration constant becomes less important as the separation in the binary is increased (see also the discussion below), but it can easily lead to errors of $10 \%$ or more for the rather close binaries considered here. A more detailed discussion on the integration constant will be given in [31].

Results. - In Fig. 2 we show the evolution of the recoil velocity for the 5 binaries considered, with the dashed lines referring to the gauge-invariant extraction and the solid lines to the one using $\Psi_{4}$; both quantities are extracted at $50 M$, which we have found to be the smallest needed distance for consistent results.

A number of interesting features are worth remarking. First, all of the curves show a monotonic growth as a result of a suitable choice for the integration constant. Second, the two types of measurements agree to very good precision. Third, binaries that have a spin ratio closer to zero

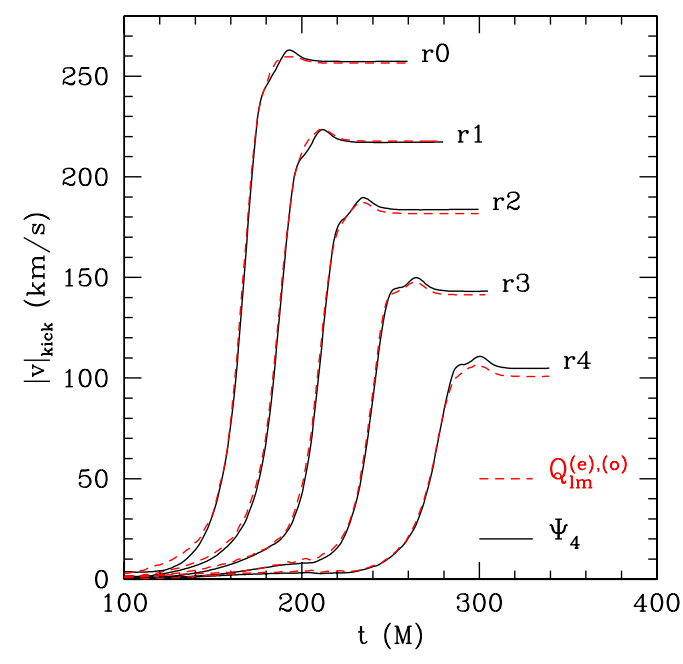

FIG. 2 (color online). Recoil velocity as function of time for the sequence of runs, i.e., from $r 0$ with $-a_{1}=a_{2}=0.586$ to $r 4$ with $a_{1}=0, a_{2}=0.586$. Note that the merger is delayed for smaller values of $\left|a_{1}\right|$.

merge progressively later. In the case of the binaries considered here this effect is partially masked by the fact that the binary $r 0$ is at a smaller separation than $r 4$ (cf. Table I). Bearing this in mind, it is, however, apparent that the growth rate of the kick velocity (and hence the rapidity of the inspiral) increases with the asymmetry in the spins. Fourth, increasing the initial separation for a binary with $a_{1} / a_{2}=-1$ does not change significantly the integration constant chosen for $r 0$, thus indicating that the kick estimate for the latter is robust. Finally, as in unequal-mass binaries, the largest contribution to the kick comes from the final parts of the inspiral and is dominated by the last orbit. However, unlike equal-mass binaries, the postmerger evolution of the kick velocity is not modified substantially by the quasinormal mode ringing (cf. Figs. 1 and 2 ), with the final kick velocity being only slightly smaller than the maximum one reached during the evolution.

As predicted by the PN expression (1), the final velocities shown in Fig. 2 exhibit a linear dependence with the spin ratio, and this is shown in Fig. 3, which reports the asymptotic kick velocities when measured with $\Psi_{4}$ (open circles) or with the gauge-invariant perturbations (stars). Also indicated are the error bars which include errors from the determination of the integration constants, from the dependence of the waveforms on the extraction radii, and from the truncation error.

The data points in Fig. 3 are not the only ones available and indeed a binary system with $a_{1} / a_{2}=1$ is bound to produce a zero kick velocity. The dashed lines in Fig. 3 represent a linear fit of all the data when the point at $a_{1} / a_{2}=1$ is given an infinite weight to account that $|v|_{\text {kick }}=0$ when $a_{1}=a_{2}$ (short-dashed line for $\Psi_{4}$ and long-dashed line for $Q_{\ell m}^{(e, o)}$ ). These lines are only indicative and bear a physical significance only if the linear dependence should hold for all the possible values of the spin ratio. 


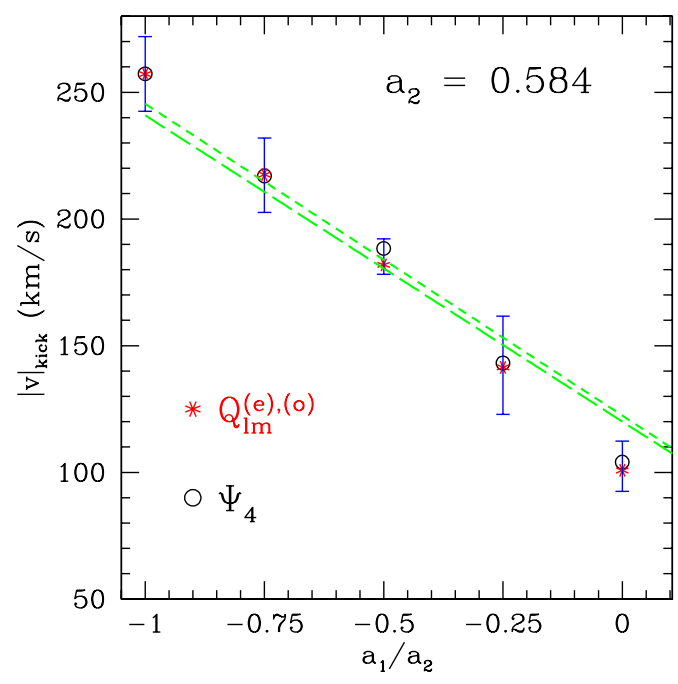

FIG. 3 (color online). Kick velocities and error bars for different spin ratios. The dashed lines show a linear fit of all the data when the point at $a_{1} / a_{2}=1$ is given an infinite weight since $|v|_{\text {kick }}=0$ for $a_{1}=a_{2}$.

Conclusions. - We have calculated the recoil velocity from the inspiral and merger of binary-black-hole systems with equal masses but unequal spins. To increase the accuracy and remove systematic errors, we have performed our measurements using both the Newman-Penrose quantity $\Psi_{4}$ as well as gauge-invariant metric perturbations of a Schwarzschild spacetime. The two methods agree to very good precision and indicate that the recoil velocity produced by spin asymmetries can be considerably larger than that expected from asymmetries in the masses. More specifically, we have found a maximum recoil velocity of $257 \pm 15 \mathrm{~km} / \mathrm{s}$ for a system having a spin ratio $a_{1} / a_{2}=$ -1 and $a_{2}=0.584$. In addition, a linear scaling holds between the kick velocity and the spin ratio, thus qualitatively confirming the PN expectation. A linear extrapolation to the case of extremal black holes, i.e., $a_{1}=1=-a_{2}$, suggests a maximal value for the kick velocity from unequal spin, but equal-mass binaries of $\simeq 440 \mathrm{~km} / \mathrm{s}$. Such a recoil velocity would be more than twice that produced by nonspinning but unequal-mass binaries.

At the time of the submission of this Letter a number of complementary studies have also been submitted [32-34], which support the results found here as well as examine nonaligned spin configurations.

It is a pleasure to thank Alessandro Nagar Bernard Schutz, Ed Seidel, and Ryoji Takahashi for useful discussions. The computations were performed at AEI, LSU/ LONI, NERSC, and at NCSA (Grant No. MCA02N014).
This work was also supported by the DFG (Grant No. SFB TR/7).

[1] F. Pretorius, Phys. Rev. Lett. 95, 121101 (2005).

[2] M. Campanelli et al., Phys. Rev. Lett. 96, 111101 (2006).

[3] J. G. Baker et al., Phys. Rev. Lett. 96, 111102 (2006).

[4] F. Pretorius, Classical Quantum Gravity 23, S529 (2006).

[5] J. G. Baker et al., Phys. Rev. D 73, 104002 (2006).

[6] M. Campanelli, C. O. Lousto, and Y. Zlochower, Phys. Rev. D 73, 061501(R) (2006).

[7] J. G. Baker et al., Phys. Rev. D 75, 124024 (2007).

[8] J. G. Baker et al., Astrophys. J. 653, L93 (2006).

[9] L. Baiotti and L. Rezzolla, Phys. Rev. Lett. 97, 141101 (2006).

[10] P. Diener et al., Phys. Rev. Lett. 96, 121101 (2006).

[11] J. A. Gonźalez et al., Phys. Rev. Lett. 98, 091101 (2007).

[12] M. Campanelli, C. O. Lousto, and Y. Zlochower, Phys. Rev. D 74, 041501 (2006).

[13] M. Campanelli, C. O. Lousto, and Y. Zlochower, Phys. Rev. D 74, 084023 (2006).

[14] M. Campanelli et al., Phys. Rev. D 75, 064030 (2007).

[15] J.G. Baker et al., Classical Quantum Gravity 24, S25 (2007).

[16] J. Thornburg et al., arXiv:gr-qc/0701038.

[17] M. Favata, S. A. Hughes, and D. E. Holz, Astrophys. J. 607, L5 (2004).

[18] T. Damour and A. Gopakumar, Phys. Rev. D 73, 124006 (2006).

[19] D. Merritt and R. D. Ekers, Science 297, 1310 (2002).

[20] L. E. Kidder, Phys. Rev. D 52, 821 (1995).

[21] M. Alcubierre et al., Phys. Rev. D 67, 084023 (2003).

[22] J. Thornburg, Classical Quantum Gravity 21, 743 (2004).

[23] E. Schnetter, S.H. Hawley, and I. Hawke, Classical Quantum Gravity 21, 1465 (2004).

[24] S. Brandt and B. Brügmann, Phys. Rev. Lett. 78, 3606 (1997).

[25] M. Ansorg, B. Brügmann, and W. Tichy, Phys. Rev. D 70, 064011 (2004).

[26] G. B. Cook, Phys. Rev. D 50, 5025 (1994).

[27] E. T. Newman and K.P. Tod, in General Relativity and Gravitation: One Hundred Years After the Birth of Albert Einstein, edited by A. Held (Plenum, New York, 1980), Vol. 2, pp. 1-36.

[28] M. Campanelli and C. O. Lousto, Phys. Rev. D 59, 124022 (1999).

[29] L. Rezzolla et al., Phys. Rev. D 59, 064001 (1999).

[30] A. Nagar and L. Rezzolla, Classical Quantum Gravity 22, R167 (2005); 23, 4297(E) (2006).

[31] D. Pollney et al., arXiv:0707.2559v1.

[32] F. Herrmann et al., arXiv:gr-qc/0701143.

[33] M. Campanelli et al., Phys. Rev. Lett. 98, 231102 (2007).

[34] J. A. Gonzalez et al., Phys. Rev. Lett. 98, 231101 (2007). 